\title{
Deciphering the minimum of energy of some walking technicolor models
}

\author{
A. Doff ${ }^{1}$ and A. A. Natale ${ }^{2}$ \\ ${ }^{1}$ Universidade Tecnológica Federal do Paraná - UTFPR - COMAT \\ Via do Conhecimento Km 01, 85503-390, Pato Branco - PR, Brazil \\ ${ }^{2}$ Instituto de Fúsica Teórica, UNESP - Univ. Estadual Paulista, \\ Rua Dr. Bento T. Ferraz, 271, Bloco II, 01140-070, São Paulo - SP, Brazil
}

(Dated: November 19, 2018)

\begin{abstract}
There are quasi-conformal theories, like the Minimal and Ultraminimal Technicolor models, which may break dynamically the gauge symmetry of the Standard Model and at the same time are compatible with electroweak precision data. The main characteristic of this type of models is their fermionic content in one or more higher dimensional representations, therefore it is not immediate to know which model leads to the most attractive channel or the minimum vacuum energy state. We discuss the effective potential for composite operators for these models, verifying that their vacuum energy values are different, with the Ultraminimal model having a deeper minimum of energy.
\end{abstract}

The nature of the Higgs boson is one of the most important problems in particle physics, and there are many questions that may be answered in the near future by the LHC experiments, such as: Is the Higgs boson, if it exists at all, elementary or composite, and what are the symmetries behind the Higgs mechanism. The possibility that the Higgs boson is a composite state instead of an elementary one is more akin to the phenomenon of spontaneous symmetry breaking that originated from the effective Ginzburg-Landau Lagrangian, which can be derived from the microscopic BCS theory of superconductivity describing the electron-hole interaction (or the composite state in our case). This dynamical origin of the spontaneous symmetry breaking has been discussed with the use of many models, being the most popular one the technicolor (TC) model [1].

Unfortunately we do not know the dynamics that form the scalar bound state, which should play the role of the Higgs boson in the standard model symmetry breaking. Most of the models for the spontaneous symmetry breaking of the standard model based on composite Higgs boson system depend on specific assumptions about the dynamics responsible for the bound state formation [2], and the work in this area try to find the TC dynamics dealing with the particle content of the theory, in order to obtain a technifermion self-energy that does not lead to phenomenological problems as in the scheme known as walking technicolor [3]. These are theories where the incompatibility with the experimental data has been solved, making the new strong interaction almost conformal and changing appreciably its dynamical behavior. We can obtain an almost conformal TC theory, when the fermions are in the fundamental representation, introducing a large number of TC fermions $\left(n_{F}\right)$, leading to an almost zero $\beta$ function and flat asymptotic coupling constant. The cost of such procedure may be a large S parameter [4] incompatible with the high precision electroweak measurements.

TC models with fermions in other representations than the fundamental one, like happens in the Minimal [6] (MWT) and Ultraminimal 7] (UMT) TC models, are possible viable models without conflict with the known value for the measured S parameter, which may be calculated assuming valid the perturbative expressions for such parameter. These models have some phenomenological differences [8], although their fermionic content is not totally different and it is possible to have even more extensions of this type of models [9]. They also have a different number of composite scalar particles as well as different couplings among themselves [10].

There is a striking difference between models based on fundamental or composite scalar bosons. In the case of a fundamental scalar boson we just have a scalar potential with a mass and coupling constant conveniently adjustable to provide the correct gauge symmetry breaking of the Standard Model. In the case of a composite scalar we do have a gauge theory at some energy scale with some fermionic content, and everything should be calculable in terms of these quantities, although, due to the non-perturbative aspects of the symmetry breaking, it is much more difficult to obtain precise evaluations of the physical parameters. In this work we will investigate another characteristic of these models, which is the value of the state of minimum energy (the most attractive channel), i.e. discover which model leads to the tightest bound states. This type of information can be obtained with the use of an effective potential for composite operators 5], and this is a more involved quantity to compute when the theory has fermions in several (and higher dimensional) representations, because it is not just a matter of comparing Casimir operator eigenvalues as is usually performed for a gauge group with an unique fermionic representation.

In the sequence we introduce the Minimal and Ultraminimal TC models, discuss the fermionic self-energies solutions for these models, and compute the vacuum energy with these solutions. We also use a standard walking (WT) theory (by standard we mean a theory with fermions only in the fundamental representation) to compare the different minima of energy of these quasiconformal theories. The MWT model is based on a $S U(2)$ gauge group with two adjoint fermions [6]

$$
Q_{L}^{a}=\left(\begin{array}{l}
U^{a} \\
D^{a}
\end{array}\right)_{L} \quad, \quad U_{R}^{a}, \quad D_{R}^{a}, \quad a=1,2,3
$$


where $a$ is the $S U(2)$ adjoint color index and the lefthanded fields correspond to three $\left(S U(2)_{L}\right)$ weak doublets. The UMT model is based on a two colors group with two fundamental Dirac flavors $S U(2)_{L} \times U(1)_{Y}$ charged described by [7]

$$
T_{L}=\left(\begin{array}{c}
U \\
D
\end{array}\right)_{L} \quad, \quad U_{R}, \quad D_{R}
$$

and also two adjoint Weyl fermions indicated by $\lambda^{f}$ with $f=1,2$, where these fermions are singlets under $S U(2)_{L} \times U(1)_{Y}$.

The near conformal behavior for these models can be observed looking at the zero of the two-loop $\beta\left(g^{2}\right)$ function, which is given by $\beta(g)=-\beta_{0} \frac{g^{3}}{(4 \pi)^{2}}-\beta_{1} \frac{g^{5}}{(4 \pi)^{4}}$, where $\beta_{0}=(4 \pi)^{2} b=\frac{11}{3} C_{2}(G)-\frac{4}{3} T(R) n_{F}(R)$ and $\beta_{1}=\left[\frac{34}{3} C_{2}^{2}(G)-\frac{20}{3} C_{2}(G) T(R) n_{F}-4 C_{2}(R) T(R) n_{F}\right]$. Where $C_{2}(R) I=T_{R}^{a} T_{R}^{a}, C_{2}(R) d(R)=T(R) d(G), d(R)$ is the dimension of the representation $R$ and $G$ indicates the adjoint representation. It is interesting to compare the leading term of the $\beta$ function for the different models (indicated respectively by $b_{m i}$ and $b_{u m}$, while the one of a simple walking TC theory is denoted by $b_{w}$ ). In the case of an $S U(2)$ gauge group with 8 Dirac fermions we have $b_{w}=2 / 16 \pi^{2}$, while in the Minimal walking model we obtain the same coefficient with only 2 fermions $\left(b_{w}=b_{m i}\right)$ ! The main difference among these models appears when we compute the $\mathrm{S}$ parameter whose perturbative expression (in the massless limit) is

$$
S=\frac{1}{6 \pi} \frac{n_{F}}{2} d(R) .
$$

The data requires the value of the $S$ parameter to be less than about 0.3. According to the "naive" perturbative estimate of Eq.(3) this requirement is indeed met for MWT (and also for UMT). Early models, with fermions only in the fundamental representation, needed a quite large $n_{F}$ to have a walking behavior, giving a perturbative estimate of $S$ in contradiction with data. Sannino and collaborators have extensively advocated the advantage of working with higher dimensional fermionic representations. In the Refs. [6 6 , 11, 12] walking TC models are introduced with the advantage of a small number of technifermions and in conformity with high precision standard model data.

In the Ref. 13] we introduced a very general ansatz for the technifermion self-energy that interpolates between all known forms of technifermionic self-energy. As we vary one parameter $(\alpha)$ in our ansatz for the technifermionic self-energy, we go from the standard operator product expansion (OPE) behavior of the self-energy to the one predicted by the extreme limit of a walking technicolor dynamics. The form of this ansatz is reproduced below

$$
\Sigma_{A}\left(p^{2}\right) \sim \Lambda_{T C}\left(\frac{\Lambda_{T C}^{2}}{p^{2}}\right)^{\alpha}\left[1+a \ln \left(p^{2} / \Lambda_{T C}^{2}\right)\right]^{-\beta} .
$$

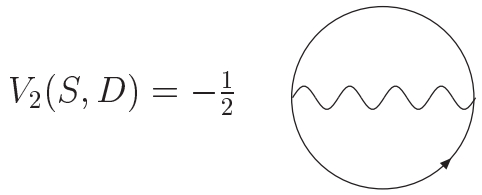

FIG. 1. Leading order contribution to $V_{2}(S, D)$.

In this expression the standard OPE behavior for $\Sigma\left(p^{2}\right)$ is obtained when $\alpha \rightarrow 1$, whereas the extreme walking technicolor solution is obtained when $\alpha \rightarrow 0$. We identify $a \equiv b g_{T C}^{2}, \beta \equiv \gamma_{T C} \cos (\alpha \pi)$ with $\gamma_{T C}=\gamma=3 c / 16 \pi^{2} b$, and $c$ is the quadratic Casimir operator given by $c=$ $\frac{1}{2}\left[C_{2}\left(R_{1}\right)+C_{2}\left(R_{1}\right)-C_{2}\left(R_{3}\right)\right] . C_{2}\left(R_{i}\right)$ are the Casimir operators for technifermions in the representations $R_{1}$ and $R_{2}$ that condensate in the representation $R_{3}, b$ is the coefficient of the $g^{3}$ term in the technicolor $\beta(g)$ function.

The TC scale $\left(\Lambda_{T C}\right)$ is related to the technicolor condensate by $\langle\bar{\psi} \psi\rangle_{T C} \approx \Lambda_{T C}^{3}$ and we can describe the TC scale in terms of measurable quantities and of group theoretical factors of the strong interaction responsible for forming the composite scalar boson. In the extreme limit of a walking technicolor dynamics we expect to have 14]

$$
\Lambda_{T C}=v\left(\frac{8 \pi^{2} a(2 \gamma-1)}{N_{T C} n_{F}}\right)^{1 / 2}
$$

where $v \sim 246 \mathrm{GeV}$ is the standard model VEV.

The effective potential for composite operators is given by the following expression [5]

$V(S, D)=-\imath \int \frac{d^{4} p}{(2 \pi)^{4}} \operatorname{Tr}\left(\ln S_{0}^{-1} S-S_{0}^{-1} S+1\right)+V_{2}(S, D)$,

where $S$ and $D$ are the complete propagators of fermions and gauge bosons and $S_{0}, D_{0}$, are the corresponding bare propagators. The function $V_{2}(S, D)$ is the sum of twoparticle irreducible vacuum diagrams, which, in the leading Hartree-Fock approximation, is depicted in the Figure (1). $V_{2}(S, D)$ can be represented analytically by

$$
\imath V_{2}(S, D)=-\frac{1}{2} \operatorname{Tr}(\Gamma S \Gamma S D),
$$

where, for simplicity, we have not written the gauge and Lorentz indices, as well as the momentum integrals and we represent the fermion proper vertex by $\Gamma$.

We want to determine the vacuum expectation value obtained with the fermionic self-energy that is given by Eq.(4), when $\alpha \approx 0$, for the (MWT), (UMT) and (WT) models. However, it is better to compute the vacuum energy density, which is given by the effective potential calculated at minimum subtracted by its perturbative part which does not contribute to dynamical mass generation [5, 15]

$$
\langle\Omega\rangle=V_{\min }(S, D)-V_{\min }\left(S_{p}, D_{p}\right),
$$

where we indicate in the expression above the perturbative counterpart of $S$ and $D$ respectively by $S_{p}, D_{p}$. 


\begin{tabular}{cccccc}
\hline \hline Model & $a$ & $\gamma_{F}$ & $\gamma_{G}$ & $n_{F}(D)$ & $n_{F}(W)$ \\
\hline WT & 0.21 & $\frac{9}{8}$ & 0 & 8 & 0 \\
MWT & 0.08 & 0 & 3 & 2 & 0 \\
UMT & 0.09 & $\frac{27}{40}$ & $\frac{18}{10}$ & 2 & 2 \\
\hline \hline
\end{tabular}

TABLE I. Values for the coefficients $a$ and $\gamma$ obtained for MWT, UMT and WT models. In this table we denoted by $n_{F}(D)$ and $n_{F}(W)$ respectively the number of Dirac and Weyl fermions.

$V_{\min }(S, D)$ is obtained substituting Eq.(44) into Eq.(6), assuming $\alpha \approx 0$. The complete fermion propagator $S$ is related to the free propagator by the equation $S^{-1}=$ $S_{0}^{-1}-\Sigma$, with $S_{0}=\imath / \not p$, and in the chiral limit $S_{p}=S_{0}$. We chose to work in the Landau gauge for simplicity and after going to Euclidean space, we find that $\Omega_{\min } \equiv\langle\Omega\rangle$ and is equal to 15 ]

$$
\Omega_{m i n}=-2 N_{T C} n_{F} \int \frac{d^{4} p}{(2 \pi)^{4}}\left[\ln \left(\frac{p^{2}+\Sigma^{2}}{p^{2}}\right)-\frac{\Sigma^{2}}{p^{2}+\Sigma^{2}}\right]
$$

We can still expand $\Omega_{\min }$ in powers of $\Sigma^{2} / p^{2}$, so that

$$
\Omega_{\min } \approx-N_{T C} n_{F} \int \frac{d^{4} p}{(2 \pi)^{4}} \frac{\Sigma^{4}}{p^{4}} .
$$

To obtain an analytical formula for the vacuum energy density we will make the substitution $x \rightarrow \frac{p^{2}}{\Lambda_{T C}^{2}}$ in the Eqs.(4) and (10), and use the following Mellin transform [16]

$$
[1+\kappa \ln x]^{-\epsilon}=\frac{1}{\Gamma(\epsilon)} \int_{0}^{\infty} d \sigma e^{-\sigma}(x)^{-\sigma \kappa} \sigma^{\epsilon-1}
$$

that will simplify considerably the calculation. In this Mellin transform we identified $\kappa=a$ and $\epsilon=4 \beta$. Then, after we substitute Eq.(44) in to (10), and perform the integration we obtain for $\alpha \approx 0[13$ ]

$$
\Omega_{\min }=-\frac{\Lambda_{T C}^{4}}{16 \pi^{2} a} \frac{N_{T C} n_{F}}{(4 \gamma-1)}\left[1-\frac{4 \alpha}{a} \frac{1}{(4 \gamma-2)}+O\left(\alpha^{2}\right) \ldots\right] .
$$

In Table I we show the values of the coefficients $a=$ $b g_{T C}^{2}$ and $\gamma_{i}$, for technifermions in the fundamental representation $(i=F)$ or adjoint $(i=G)$, obtained for the MWT model and for a conventional WT model based on $S U(2)_{T C}$.

In the case of the UMT model we cannot apply straightforwardly Eq.(5), because in this case we have two scalar composite bosons, that appear as mixed states formed by fermions in the fundamental and adjoint representation. In this model only the lightest composite boson, that is mostly formed by technifermions in the fundamental representation, is the one that couples to the particles of the Standard Model. The UMT gap equation has two contributions, one with a Casimir operator

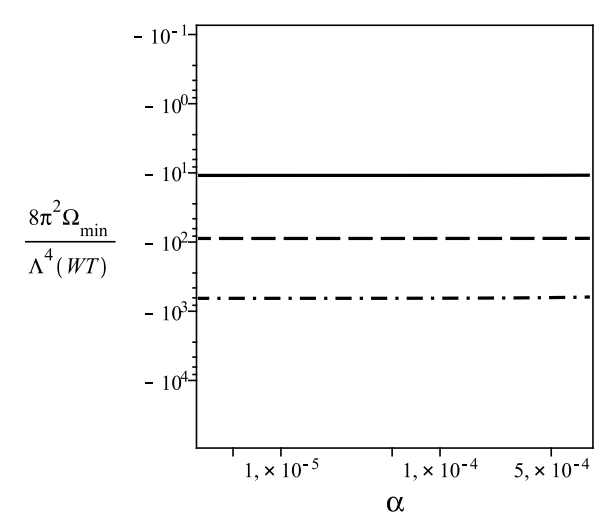

FIG. 2. Behavior of $\Phi\left(\Omega_{\min }\right) \equiv \frac{8 \pi^{2} \Omega_{\min }}{\Lambda_{T C}^{4}(W T)}$ for the WT (solid line), MWT (dashed) and UMT (dot-dashed) models plotted as a function of the $\alpha$ parameter.

for fermions in the fundamental representation and another with a different Casimir operator for fermions in the adjoint representation, while it is the same $\beta$ function that governs the running of the coupling in the two contributions.

It is opportune to remember that the gap equation lead to different chiral symmetry breaking scales when the fermions are in different representations, this has been observed, for instance, in QCD with quarks in the adjoint representation [17], where the chiral transition may be slightly different from the confinement transition, which coincides with the chiral one for fermions in the fundamental representation. We can expect that the masses and composite scalar wave functions will show a mixing but with scales quite close to the TC scale $\left(\Lambda_{T C}\right)$, therefore in the case of the UMT model we can suppose that $\Lambda_{T C}(U M T) \approx \Lambda_{T C}(M W T)$; with this approximation (i.e. we assume the same scales for the different models) we sum the contributions of the two different representations of the UMT model in order to compute Eq.(12).

It is also possible to relate the TC scale associated to the standard walking TC model $\left(\Lambda_{T C}(W T)\right)$ with the scale of the minimal model considering Eq.(15), which scale with the term between brackets

$$
\frac{\Lambda_{T C}(W T)}{\Lambda_{T C}(M W T)}=\frac{\left(\frac{a(2 \gamma-1)}{n_{F}}\right)_{W T}^{1 / 2}}{\left(\frac{a(2 \gamma-1)}{n_{F}}\right)_{M W T}^{1 / 2}} .
$$

Based on the above relation we can compute Eq.(12) for all models considering only a single scale $\left(\Lambda_{T C}(W T)\right.$, and we can define for $S U(2)_{T C}$ the following quantity

$$
\Phi\left(\Omega_{m i n}\right) \equiv \frac{8 \pi^{2} \Omega_{m i n}}{\Lambda_{T C}^{4}(W T)} .
$$

The value of Eq.(14) is plotted in Fig.(2) as a function of the $\alpha$ parameter. In this figure the solid line corresponds to $\Phi\left(\Omega_{\min }\right)$ obtained for the standard walking 
TC model, the dashed line represents the corresponding result for the MWT model whereas the dot-dashed line is the result obtained for the UMT model.

We have seen that the walking behavior can be obtained in many ways, for example assuming a large number of technifermions in the fundamental representation or considering a small number of technifermions in higher dimensional representations. In this work we consider three different models that lead to the walking behavior and take the same form for the technifermion selfenergy. However, analyzing Fig.(2) we verify that these three models have different values for the vacuum energy density. This result can be understood as follows, as in the case of QCD with quarks in higher representations of $S U(3)_{c}$ 18], technifermions in higher representations of $S U(2)_{T C}$ naturally interact more strongly than conventional technifermions and therefore lead to the deepest state of energy. These models can lead to a similar phenomenology, that in principle may be tested at the LHC, therefore, it is interesting to consider a criterion that could be used to select which of these approaches may be the most promising to promote the standard model symmetry breaking. Sannino and collaborators have extensively advocated the advantage of working with higher dimensional fermionic representations, in particular, in the refs. 6 8, 11, 12] are introduced the MWT and UMT models with a small number of technifermions and in conformity with high precision standard model data.

In this work we proposed a mechanism to select the most probable walking technicolor dynamics assuming an energy criterion. We show that the Ultraminimal walking TC models leads to a lower value for the minimum of the effective potential, or the formation of tightest bound states, with the advantage of a small number of technifermions.

\section{ACKNOWLEDGMENTS}

This research was partially supported by the Conselho Nacional de Desenvolvimento Científico e Tecnológico (CNPq).
[1] F. Sannino, "Lectures presented at the $49^{\text {th }}$ Cracow School of Theoretical Physics", hep-ph/0911.0931.

[2] C. T. Hill and E. H. Simmons, Phys. Rept. 381, 235 (2003) [Erratum-ibid. 390, 553 (2004)].

[3] B. Holdom, Phys. Rev. D24,1441 (1981);Phys. Lett.B150, 301 (1985); T. Appelquist, D. Karabali and L. C. R. Wijewardhana, Phys. Rev. Lett. 57, 957 (1986); T. Appelquist and L. C. R. Wijewardhana, Phys. Rev. D36, 568 (1987); K. Yamawaki, M. Bando and K.I. Matumoto, Phys. Rev. Lett. 56, 1335 (1986); T. Akiba and T. Yanagida, Phys. Lett. B169, 432 (1986).

[4] M. E. Peskin and T. Takeuchi, Phys. Rev. Lett. 65, 964 (1990); Phys. Rev. D 46, 381 (1992).

[5] J. M. Cornwall, R. Jackiw and E. Tomboulis, Phys. Rev. D10, 2428 (1974).

[6] R. Foadi, M. T. Frandsen, T. A. Ryttov and F. Sannino, Phys. Rev. D 76, 055005 (2007).

[7] M. Jarvinen, C. Kouvaris and F. Sannino, Phys. Rev. D 78, 115010 (2008).

[8] M. Jarvinen, T. A. Ryttov and F. Sannino, Phys. Rev. D 79, 095008 (2009); A. Belyaev, R. Foadi, M. T. Frandsen, M. Jarvinen, F. Sannino and A. Pukhov, Phys. Rev. D
79, 035006 (2009); T. A. Ryttov and F. Sannino, Phys. Rev. D 78, 115010 2008; R. Foadi and F. Sannino, Phys. Rev. D 78, 037701 (2008).

[9] M. Antola, S. Di Chiara, F. Sannino and K. Tuominen, e-Print: arXiv:1001.2040 [hep-ph].

[10] A. Doff and A. A. Natale, hep-ph/0912.1003.

[11] R. Foadi, M. T. Frandsen, T. A. Ryttov and F. Sannino, Phys. Rev. D 76, 055005 (2007)

[12] T. A. Ryttov and F. Sannino, Phys. Rev. D 78, 115010 (2008).

[13] A. Doff and A. A. Natale, Phys. Lett. B537, 275 (2002).

[14] A. Doff and A. A. Natale, Phys. Lett. B677, 301 (2009).

[15] P. Castorina and S.-Y.Pi, Phys. Rev. D31, 411 (1985); V. P. Gusynin and Yu. A. Sitenko, Z. Phys. C29, 547 (1985).

[16] J. M. Cornwall and R. C. Shellard, Phys. Rev. D18, 1216 (1978).

[17] J. M. Cornwall, talk at the symposium Approaches to Quantum Chromodynamics, Oberwöls, September (2008), hep-ph/0812.0395.

[18] W. J. Marciano, Phys. Rev. D21, 2425 (1980). 